\title{
Epidural hematoma following epidural catheter removal after a single dose of clopidogrel
}

\author{
Micheline Nguyen, MD, FRCPC $\cdot$ Stephan R. Williams, MD, \\ PhD · Jean-François Gagné, MD $\mathbb{B}$
}

Received: 18 June 2019/Revised: 10 September 2019/Accepted: 11 September 2019/Published online: 3 October 2019

(C) Canadian Anesthesiologists' Society 2019

\section{To the Editor,}

The American Society for Regional Anesthesia (ASRA) guidelines attempt to balance the risk of withholding antiplatelet medication against that of epidural hematoma after neuraxial anesthesia. ${ }^{1}$ We present a case of epidural hematoma occurring after epidural catheter removal in a patient who had received only a single postoperative dose of clopidogrel.

An elderly patient with colorectal cancer and a past medical history of stable angina, chronic back pain, mild chronic renal insufficiency, past abdominal aortic aneurysm repair, and diabetes mellitus presented for open lower anterior resection. The patient was on clopidogrel 75 $\mathrm{mg} \cdot \mathrm{day}^{-1}$ (withheld seven days prior to surgery) and aspirin $81 \quad \mathrm{mg} \cdot \mathrm{day}^{-1} \quad$ (which was continued perioperatively). Before induction of general anesthesia, a T9-T10 thoracic epidural catheter (Flextip Plus ${ }^{\mathrm{TM}}$; Teleflex Medical Canada, Oshawa, ON, Canada) was placed on the first pass using a standard technique and a $17 \mathrm{G}$ Perisafe ${ }^{\mathrm{TM}}$ Tuohy needle (BD-Canada, Mississauga, ON, Canada). Anesthesia and surgery proceeded uneventfully.

On postoperative day (POD) 3, the patient was noted to have a persistent troponin elevation and after cardiologic consultation, clopidogrel $75 \mathrm{mg}$ (without a loading dose) was administered, and manually charted, therefore bypassing the electronic medical record surveillance. The Acute Pain Service was subsequently advised of the need for antiplatelet therapy, and proceeded to remove the

M. Nguyen, MD, FRCPC · S. R. Williams, MD, PhD .

J.-F. Gagné, MD ( ()

Department of Anesthesiology and Pain Medicine, Centre

hospitalier de l'Université de Montréal (CHUM), Montréal, QC,

Canada

e-mail: jean-francois.gagne.5@umontreal.ca epidural catheter, after being assured by the nursing staff and verifying in the electronic medical record that no clopidogrel administration had occurred (though the $75 \mathrm{mg}$ dose had in fact been administered $30 \mathrm{hr}$ before). The last dose of subcutaneous heparin 5,000 units had been given eight hours prior to catheter removal; coagulation laboratory values (international normalized ratio, 0.98; platelet count, $225 \times 10^{9} \cdot \mathrm{L}^{-1}$ ) were normal. During catheter removal, excessive bleeding was noted through the catheter and at the insertion site. The patient immediately complained of sudden and intense back pain coinciding with catheter removal.

Despite the pain, the initial neurologic exam was normal. Upon further scrutiny of the patient's medical record, the manually charted dose of clopidogrel administered before catheter removal was discovered. The consulting neurosurgeon recommended stopping all anticoagulants and administration of an adult dose of platelets. It was decided to proceed with urgent imaging only if other neurologic symptoms occurred. On POD 4, the patient had no new neurologic symptoms, but the mild back pain persisted. Thus, a computed tomography scan of the spine was done which showed an epidural hematoma 7 $\mathrm{mm}$ in diameter extending from T9 to L2 compressing the spinal cord. The patient did not develop any neurologic symptoms and magnetic resonance imaging a month later showed regression of the hematoma with less spinal cord compression (Figure).

Thienopyridines such as clopidogrel are often used in the treatment of coronary, vascular, and cerebrovascular events. The most recent guidelines presented by the ASRA $^{1}$ suggest that an epidural catheter could be maintained for one to two days when starting clopidogrel treatment without a loading dose, since steady states of clopidogrel are only achieved after seven days. In this case, 


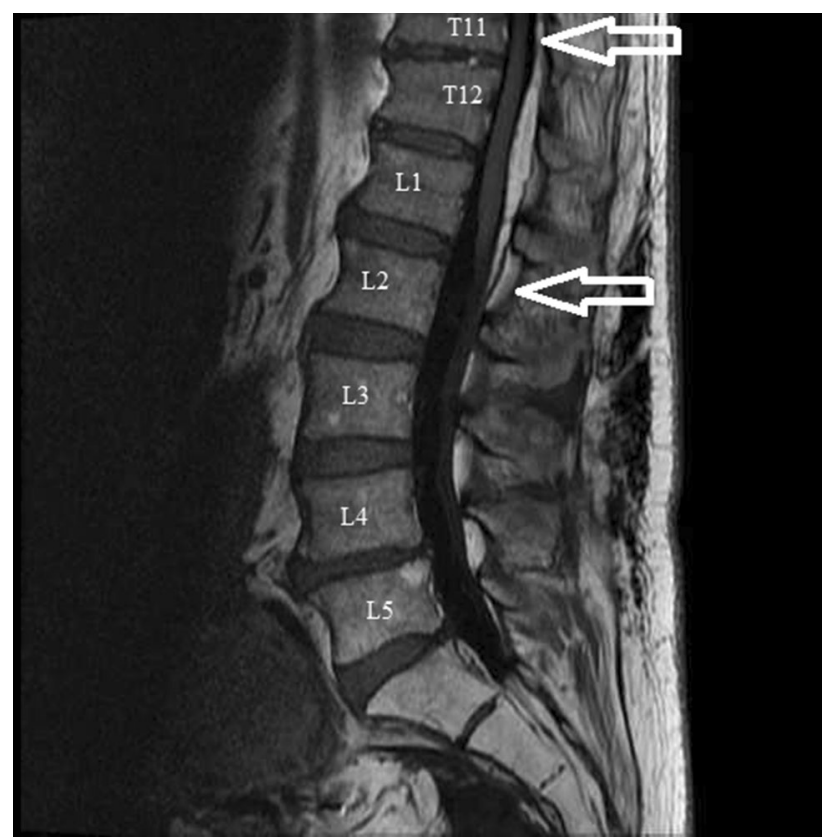

Figure A T1-weighted magnetic resonance imaging (MRI) scan done one month after the conservative (non-surgical) management of a patient who developed an epidural hematoma following T9-T10 epidural catheter removal. The MRI shows a regression of the hematoma (white arrows) that is seen from T11 to L1.

epidural hematoma occurred in a patient who had received a single dose of clopidogrel $30 \mathrm{hr}$ before epidural catheter removal, in agreement with new ASRA guidelines regarding the combination of antiplatelet medication and neuraxial anesthesia techniques. Guidelines attempting to manage the infrequent but potentially devastating risk of epidural hematoma rely in part on case reports of adverse events such as the one presented above. We report the present case to help clinicians in the complex case-by-case evaluation of the risks and benefits of initiating antiplatelet therapy and assessing concurrent medications that may have an effect on coagulation with an epidural catheter in place.

Conflicts of interest None declared.

Funding The authors have no sources of funding to declare for this manuscript.

Editorial responsibility This submission was handled by Dr. Hilary P. Grocott, Editor-in-Chief, Canadian Journal of Anesthesia.

\section{Reference}

1. Horlocker TT, Vandermeuelen E, Kopp SL, Gogarten W, Leffert $L R$, Benzon HT. Regional Anesthesia in the Patient Receiving Antithrombotic or Thrombolytic Therapy: American Society of Regional Anesthesia and Pain Medicine Evidence-Based Guidelines (Fourth Edition). Reg Anesth Pain Med 2018; 43: 263-309.

Publisher's Note Springer Nature remains neutral with regard to jurisdictional claims in published maps and institutional affiliations. 